\title{
A QUADRATIC PROGRAMMING FRAMEWORK FOR CONSTRAINED AND ROBUST JET ENGINE HEALTH MONITORING
}

\section{S. Borguet and O. Léonard}

Kalman filters are largely used in the jet engine community for condition monitoring purpose. This algorithm gives a good estimate of the engine condition provided that the residuals between the model prediction and the measurements are zero-mean, Gaussian random variables. In the case of sensor faults, this assumption does not hold anymore and consequently, the diagnosis is spoiled. This contribution presents a recursive estimation algorithm based on a Quadratic Programming (QP) formulation which provides robustness against sensor faults and allows constraints on the health parameters to be specified. The improvements in estimation accuracy brought by this new algorithm are illustrated on a series of typical test-cases that may be encountered on current turbofan engines.

\section{NOMENCLATURE}

$(\hat{\bullet})$

$(\cdot)^{-}$

A8IMP nozzle exit area (nominal value: $1.4147 \mathrm{~m}^{2}$ )

b

estimated value

hpc

hpt

$k$

prior value

$\mathrm{KF}$

the sensor fault vector

lpc

high-pressure compressor

high-pressure turbine

discrete time index

lpt

Kalman Filter

$p_{i}^{0}$

low-pressure compressor

low-pressure turbine

$\mathrm{QP}$

total pressure at station $i$

SEi efficiency scaler at station $i$ (nominal value: 1.0 )

SFDI Sensor Fault Detection and Isolation

SWiR flow capacity scaler at station $i$ (nominal value: 1.0 )

$T_{i}^{0} \quad$ total temperature at station $i$ 


$\begin{array}{ll}\mathbf{u} & \text { operating point parameters } \\ \mathbf{w} & \text { health parameters } \\ \mathbf{y} & \text { observed measurements } \\ \epsilon & \text { measurement noise vector } \\ \mathcal{N}(\mathbf{m}, \mathbf{R}) & \begin{array}{l}\text { a Gaussian probability distribution with mean } \mathbf{m} \text { and covari- } \\ \text { ance matrix } \mathbf{R}\end{array} \\ \sigma & \text { nominal standard deviation of the sensors } \\ \omega & \text { process noise vector }\end{array}$

\section{INTRODUCTION}

Over the last decades, significant research efforts have been undertaken to improve schedule of the maintenance actions of jet engines. Many improvements can be brought by the application of condition-based maintenance where the actions are decided based on the actual health condition of the engine rather than on the sole basis of the number of operating hours. Of course, conditionbased maintenance is only achievable provided that the health of the engine is accurately monitored which involves the recording and the analysis of engine performances over a period of time.

Based on this trend analysis, significant changes in the mechanical conditions of the engine - degradations - can be detected which provides a means of deciding which maintenance action has to be carried out. Condition-based maintenance is intended to improve engine operability, to provide a longer lifetime and a better availability, but also to lower the cost of maintenance through a better prediction of overhaul actions. This context explains the stronger emphasis on turbine engine performance monitoring given over the last years by the engine manufacturers.

In the framework of performance monitoring applied to condition-based maintenance, degradations of interest are those that are neither directly measurable nor detectable without a deep inspection of the engine. Degradations are split up into progressive wear due to normal operations (erosion, rubbing wear, corrosion, fouling, mechanical wear) and damages due to accidental events (turbine overheatings, heavy landings, foreign object digestions). While the former vary very slowly, the latter are short events involving a brutal drift in engine performances.

These degradations are detected through the analysis of observable performance parameters, the set of which depends on the type and the mission of the engine. Engine instruments are used to monitor the operation of the engine and convey vital information on rotational spool speeds, engine pressure ratio (EPR) and exhaust gas temperature (EGT). Fuel flow as well as oil quantity, pressure, temperature, and vibration indicators are also available on-board. 


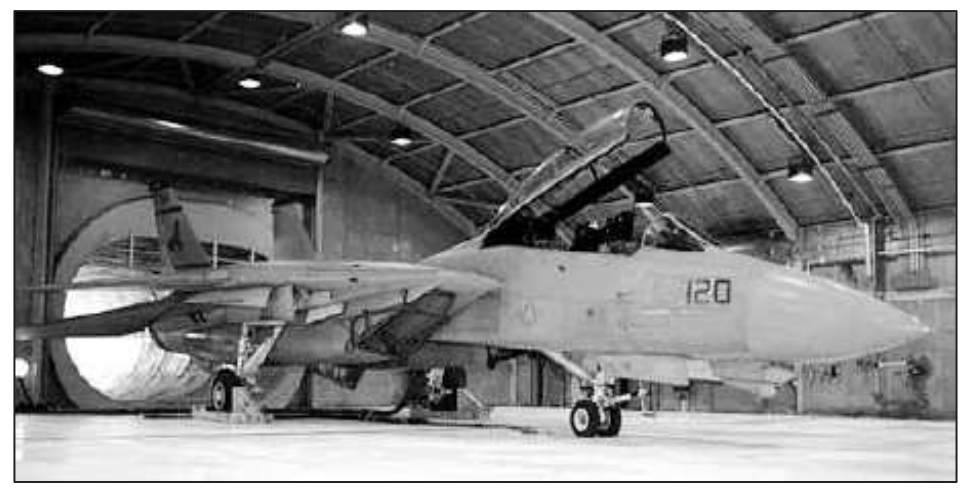

Figure 1 F14 fighter aircraft at performance test

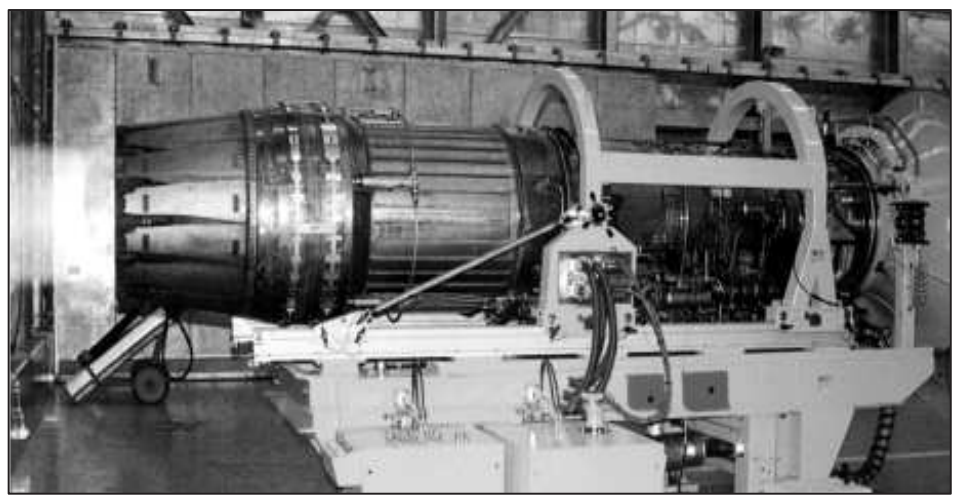

Figure 2 F100-PW100 military engine at test bench

Special devices are added to detect indications of trouble which may not be revealed by the engine instruments. However, as actual sensors remain intrusive, their integration is always limited by cost, weight, or other constraints due to engine operation and aircraft turbine-engine special instrumentation is usually kept to a minimum.

For on-wing maintenance, the test is carried out while keeping the engine installed in the aircraft (Fig. 1). While this configuration is sufficient to decide whether the engine is acceptable or not, it turns out to be insufficient to achieve a complete diagnostic (detect and isolate an engine fault) because of the lack of engine instruments available on-board. To overcome the lack of on-board instruments, the engine is unmounted from the aircraft for testing in a specific 
ground test cell (Fig. 2). The most striking argument which justifies specific test cells is the availability of the thrust measurement which is the most meaningfull quantity of interest for an aircraft turbine engine and which cannot be measured on-board because it would require the engine to be freely-mounted. Depending on the situations, other instruments may also be installed (e.g., pressures, temperatures, air mass flow rates, etc.) increasing the information available for a test bench diagnostic.

Even if ground testing is well adapted to the solving of the diagnosis problem, it must be kept to a minimum for economic reasons and for noise problems. It is often only carried out after engine installation or for overhaul and cannot provide an early detection of engine faults. With the advent of condition-based maintenance and the steadily rising emphasis on the underlying performance assessment, researches on on-board performance monitoring are encouraged, whose purpose is to detect, isolate, and quantify, at the earliest possible stage, a specific component performance degradation.

\subsection{Gas Path Analysis}

In this contribution, Module Performance Analysis, also known as Gas Path Analysis (GPA), is considered. Its purpose is to assess the changes in engine module performance on the basis of measurements collected along the gas path of the engine [1]. This approach is intended to be relative, in the sense that it is based on a modification of the gas turbine performance rather than on the determination of their absolute value. This approach allows to track the evolution of a particular engine relative to its initial performance or relative to some nominal performance averaged on a number of similar engines. The GPA approach is illustrated on Fig. 3, and its principles are the following:

- the state of the components (fan, lpc, hpc, hpt, lpt, nozzle) can be represented by a set of performance indicators called health parameters noted $\mathbf{w}$ which are typically correcting factors on their efficiency and flow capacity;

- a degradation (progressive or accidental) encountered by the engine components induce a modification of their performance, which, in turn, induces a drift of the global performance of the engine (thrust, fuel consumption) and of some measurements noted $\mathbf{y}$ collected along the gas path (temperatures, pressures, mass flows, shaft speeds); and

- it is possible to derive a physical or mathematical model of the engine that relates the variation of the health parameters $\mathbf{w}$ to the drift of the quantities $\mathbf{y}$ measured along the gas path. 


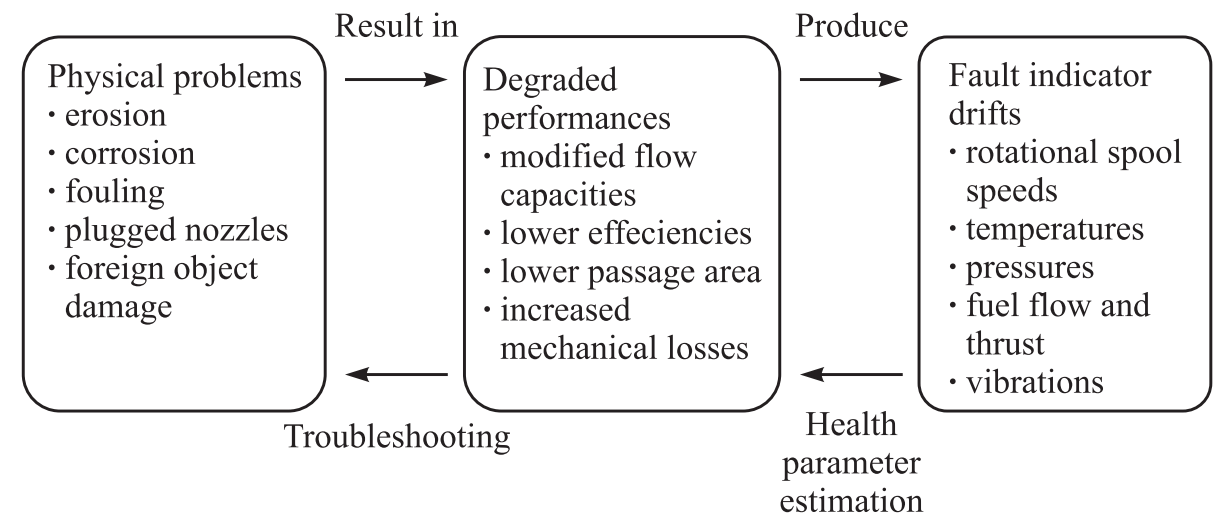

Figure 3 The GPA approach of gas turbine diagnostic

As the GPA approach to diagnosis considers that the drifts of the observable signals from reference (healthy) values is described by a function of the health parameters $\mathbf{w}$, the latter can be estimated by finding the value $\hat{\mathbf{w}}$ which minimizes the distance between the model prediction $\hat{\mathbf{y}}_{w}$ and the measured value $\mathbf{y}$. Such a problem can be stated by the resolution of the following minimization problem:

$$
\hat{\mathbf{w}}=\arg \min _{\mathbf{w}}\left\{\rho_{k}\left(\mathbf{y}-\hat{\mathbf{y}}_{w}\right)\right\}
$$

where the form of the loss function $\rho_{k}(\cdot)$ must be specified. The diagnosis problem can be thought of solving the inverse problem of prediction (see Fig. 3, the health parameter estimation arrow). This inverse problem will be solved using a Kalman filter, as it will be shown in the next section.

\subsection{Robustness Against Sensor Faults}

Besides component degradations, sensor faults may occur too. Loosely speaking, a sensor fault is a data generated by a device whose behavior does no longer follow the manufacturer characteristics. Instrumentation faults can be classified as systematic errors, such as biases and drifts, or as impulsive noise which are random "spikes" in the measured data. These instrumentation faults can spoil the estimation of the engine condition. It is therefore mandatory to make diagnosis tools robust against sensor faults.

A variety of solutions have been studied in the community, as briefly summarized below:

- data cleaning / filtering: it is actually a preprocessing of the measurements, the purpose of which is to remove aberrant values from the samples before they are used in the monitoring algorithm $[2,3]$; 
- instrumental variables: this second solution consists in treating sensor faults symmetrically to component faults. To this end, parameters intended to model sensor faults are introduced in the diagnosis problem as additional unknowns to be estimated together with the health parameters $[4-6]$;

- banks of Kalman filters have also been applied to cope with sensor faults: each filter uses all sensors but one to estimate the health parameters. The filter that does not rely on the faulty sensor is the only achieving an accurate estimation $[7,8]$; and

- robust estimation techniques: the concept is to include robustness against sensor faults within the estimation algorithm itself. The robustness is obtained by replacing the least-squares criterion by another one (to be defined later), less sensitive to outliers [9-11]. In short, an outlier can be defined as an observation that lies outside the overall pattern of a given distribution.

In the present contribution, the development of a new algorithm for performance monitoring based on robust estimation is reported. One of the drawbacks associated with robust estimation is that the estimation problem becomes nonlinear even in the case of a linear system model which increases the computational load. However, it has been shown that the robust estimation problem can be transformed into a QP problem [12] for which efficient solvers are available. The authors have reported in a previous publication the development of a robust algorithm for performance monitoring using this QP formulation [13], and have shown the benefit in terms of stability and accuracy brought by this robust diagnosis tool.

Sometimes, additional information about the health parameters is available, such as constraints based on physical considerations (e.g., aerodynamic efficiencies are not expected to improve over time). Yet, they are generally disregarded in the estimation process because they do not fit into the structure of the Kalman filter. Some research $[14,15]$ has been dedicated to this issue and has shown improved accuracy on the estimated quantities at the price of a tedious inclusion of the constraints. The QP approach allows integration of these constraints in a relatively easy way.

In the light of these considerations, the present contribution reports the formulation of a recursive QP algorithm for robust and constrained engine performance monitoring. The novelty lies in the natural inclusion of constraints on the health parameters in the estimation problem and in a wise recursive implementation. The benefit in terms of stability and accuracy brought by this new methodology is illustrated for several sensor faults that may be encountered on a jet engine. 


\section{STATEMENT OF THE PROBLEM}

The scope of this section is to present the maximum a posteriori approach used to solve the diagnostic problem as well as the corresponding Kalman filter. The sensitivity of the Kalman filter with respect to outliers is underlined, and the approach to turn the robust estimation problem into a $\mathrm{QP}$ one is presented. Finally, the inclusion of constraints on the health parameters and the structure of the resulting algorithm are discussed.

\subsection{Simulation Model}

One of the master pieces of the GPA approach is a simulation model of the engine. These are generally nonlinear aerothermodynamic models based on mass, energy and momentum conservation laws applied to the engine flowpath. Equation

$$
\mathbf{y}_{k}=\mathcal{G}\left(\mathbf{u}_{k}, \mathbf{w}_{k}\right)+\epsilon_{k}
$$

represents such an engine model where $k$ is a discrete time index; $\mathbf{u}_{k}$ are the parameters defining the operating point of the engine (e.g., fuel flow, altitude, Mach number); $\mathbf{w}_{k}$ are the aforementioned health parameters; and $\mathbf{y}_{k}$ are the gas path measurements. A random variable $\epsilon_{k} \in \mathcal{N}\left(\mathbf{0}, \mathbf{R}_{\mathbf{y}}\right)$ which accounts for sensor inaccuracies is added to the deterministic part $\mathcal{G}(\cdot)$ of the model.

\subsection{The Maximum a Posteriori Approach}

The maximum a posteriori approach is based on a Bayesian view of the diagnostic problem and allows one to answer the following question: According to the measurements $\mathbf{y}_{k}$, what is the confidence that $\mathbf{w}_{k}$ falls in a certain range? In this Bayesian view, not only the measurements but also the health parameters $\mathbf{w}_{k}$ to be determined are considered as Gaussian random variables*.

Within this framework, the estimated health parameters are obtained by minimizing the following objective function:

$$
\mathcal{J}\left(\mathbf{w}_{k}\right)=\frac{1}{2}\left(\mathbf{w}_{k}-\hat{\mathbf{w}}_{k}^{-}\right)^{T}\left(\mathrm{P}_{\mathbf{w}, k}^{-}\right)^{-1}\left(\mathbf{w}_{k}-\hat{\mathbf{w}}_{k}^{-}\right)+\frac{1}{2} \mathbf{r}_{k}^{T} \mathbf{R}_{\mathbf{y}}^{-1} \mathbf{r}_{k}
$$

The first term in the right-hand side of Eq. (2) forces the identified parameters to lie in a neighborhood of prior values $\hat{\mathbf{w}}_{k}^{-}$. The prior covariance matrix $\mathbf{P}_{\mathbf{w}, k}^{-}$ specifies the shape of this region and summarizes the information contained in the measurement history up to time $k-1$. The second term expresses a

\footnotetext{
${ }^{*}$ Statistically, they are hence thoroughly defined by their mean and covariance matrix.
} 
weighted-least-squares criterion. The residuals $\mathbf{r}_{k}$ represent the distance between the parameters measured along the gas path and their prediction by the engine model. The residuals are approximated with a semilinear approach:

$$
\mathbf{r}_{k}=\mathbf{y}_{k}-\hat{\mathbf{y}}_{k}=\mathbf{y}_{k}-\left[\hat{\mathbf{y}}_{k}^{-}+\mathbf{G}\left(\mathbf{w}_{k}-\hat{\mathbf{w}}_{k}^{-}\right)\right]=\hat{\mathbf{r}}_{k}^{-}-\mathbf{G}\left(\mathbf{w}_{k}-\hat{\mathbf{w}}_{k}^{-}\right)
$$

where

$$
\begin{aligned}
\hat{\mathbf{y}}_{k}^{-} & =\mathcal{G}\left(\mathbf{u}_{k}, \hat{\mathbf{w}}_{k}^{-}\right) \\
\mathbf{G} & =\left.\frac{\partial}{\partial \mathbf{w}_{k}} \mathcal{G}\left(\mathbf{u}_{k}, \mathbf{w}_{k}\right)\right|_{\mathbf{w}_{k}=\mathbf{w}^{h l}}
\end{aligned}
$$

are respectively the a priori prediction of the measurements and the Jacobian matrix of the engine model around nominal values $\mathbf{w}^{h l}$ of the health parameters. The residuals are weighted with a covariance matrix $\mathbf{R}_{\mathbf{r}}$ that takes into account the fact that both the measurements and the operating conditions are sensed and hence noisy:

$$
\mathbf{R}_{\mathbf{r}}=\mathbf{R}_{\mathbf{y}}+\mathbf{H}^{T} \mathbf{R}_{\mathbf{u}} \mathbf{H}
$$

where

$$
\mathbf{H}=\frac{\partial}{\partial \mathbf{u}_{k}} \mathcal{G}\left(\mathbf{u}_{k}, \mathbf{w}_{k}\right)
$$

is the influence matrix between the operating conditions and the measurements and $\mathbf{R}_{\mathbf{u}}$ is the covariance matrix of the noise on the measured operating conditions.

To generate the a priori values of the health parameter distribution (i.e., mean $\hat{\mathbf{w}}_{k}^{-}$and covariance $\mathbf{P}_{\mathbf{w}, k}^{-}$), a model describing the temporal evolution of the parameters must be supplied as well. Generally, few information is available about the way the engine degrades which motivates the choice of a random walk model:

$$
\mathbf{w}_{k}=\mathbf{w}_{k-1}+\omega_{k}
$$

where $\omega_{k} \in \mathcal{N}\left(\mathbf{0}, \mathbf{Q}_{k}\right)$ is the so-called process noise that provides some adaptability to track a time-evolving fault.

The update rule for the health parameters is found by deriving Eq. (2) with respect to $\mathbf{w}_{k}$ and setting it to zero. The following equation is obtained:

$$
\left(\mathbf{P}_{\mathbf{w}, k}^{-}\right)^{-1}\left(\mathbf{w}_{k}-\hat{\mathbf{w}}_{k}^{-}\right)-\mathbf{G}_{k}^{T} \mathbf{R}_{\mathbf{y}}^{-1} \mathbf{r}_{k}=0 .
$$

Substituting Eq. (3) into Eq. (5) leads to the following result:

$$
\left[\left(\mathbf{P}_{\mathbf{w}, k}^{-}\right)^{-1}+\mathbf{G}^{T} \mathbf{R}_{\mathbf{r}}^{-1} \mathbf{G}\right]\left(\mathbf{w}_{k}-\hat{\mathbf{w}}_{k}^{-}\right)-\mathbf{G}^{T} \mathbf{R}_{\mathbf{r}}^{-1} \hat{\mathbf{r}}_{k}^{-}=0 .
$$

From this relation, an estimation of the health parameters is found:

$$
\hat{\mathbf{w}}_{k}=\hat{\mathbf{w}}_{k}^{-}+\left[\left(\mathbf{P}_{\mathbf{w}, k}^{-}\right)^{-1}+\mathbf{G}^{T} \mathbf{R}_{\mathbf{r}}^{-1} \mathbf{G}\right]^{-1} \mathbf{G}^{T} \mathbf{R}_{\mathbf{r}}^{-1} \hat{\mathbf{r}}_{k}^{-}
$$


which provides an update rule for the health parameters:

$$
\hat{\mathbf{w}}_{k}=\hat{\mathbf{w}}_{k}^{-}+\mathbf{K} \hat{\mathbf{r}}_{k}^{-}
$$

in which the matrix $\mathbf{K}$ is defined as

$$
\mathbf{K}=\left[\left(\mathbf{P}_{\mathrm{w}, k}^{-}\right)^{-1}+\mathbf{G}^{T} \mathbf{R}_{\mathbf{r}}^{-1} \mathbf{G}\right]^{-1} \mathbf{G}^{T} \mathbf{R}_{\mathbf{r}}^{-1}
$$

The update rule is based on a prediction step (the a priori knowledge $\hat{\mathbf{w}}_{k}^{-}$) followed by a correction step involving the distance between the measured values and those predicted by the engine model, as well as the distance between the a priori values of the health parameters and their reference (healthy) values.

\subsection{Kalman Filter Implementation}

The semilinear approach and the resulting prediction-correction structure developed in the previous section can be naturally implemented through a Kalman filter [16]. A number of researchers have used this recursive algorithm in order to assess the health parameters.

The matrix $\mathbf{K}$ defined by the relation (7) will be the gain matrix of the Kalman filter. Unfortunately, the calculation of $\mathbf{K}$ involves three matrix inversions which is costly in terms of calculation effort. Using the following lemma:

$$
\left(\mathbf{A}-\mathbf{B D}^{-1} \mathbf{C}\right)^{-1} \mathbf{B D}^{-1}=\mathbf{A}^{-1} \mathbf{B}\left(\mathbf{D}-\mathbf{C A}^{-1} \mathbf{B}\right)^{-1}
$$

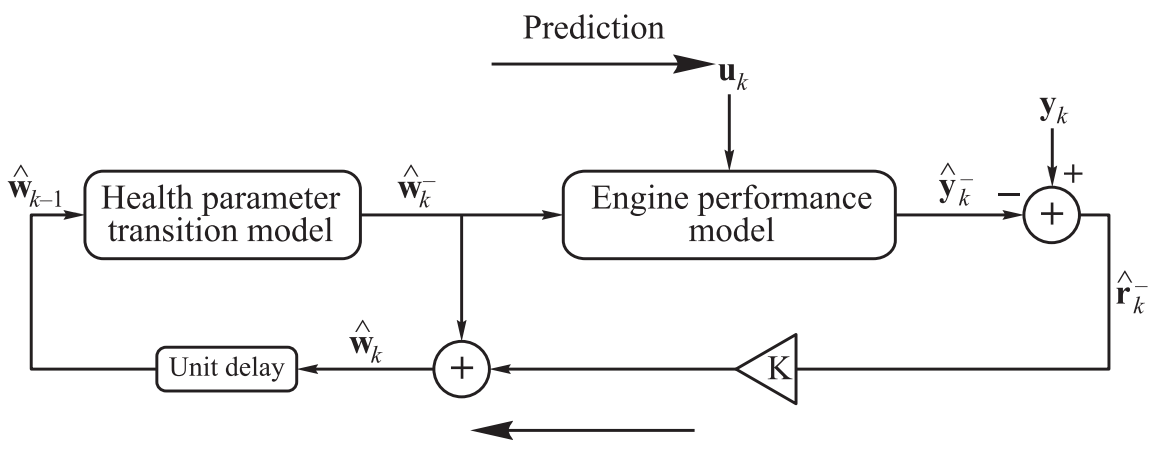

Correction

Figure 4 Kalman filter logic for the sequential estimation of the health parameters 


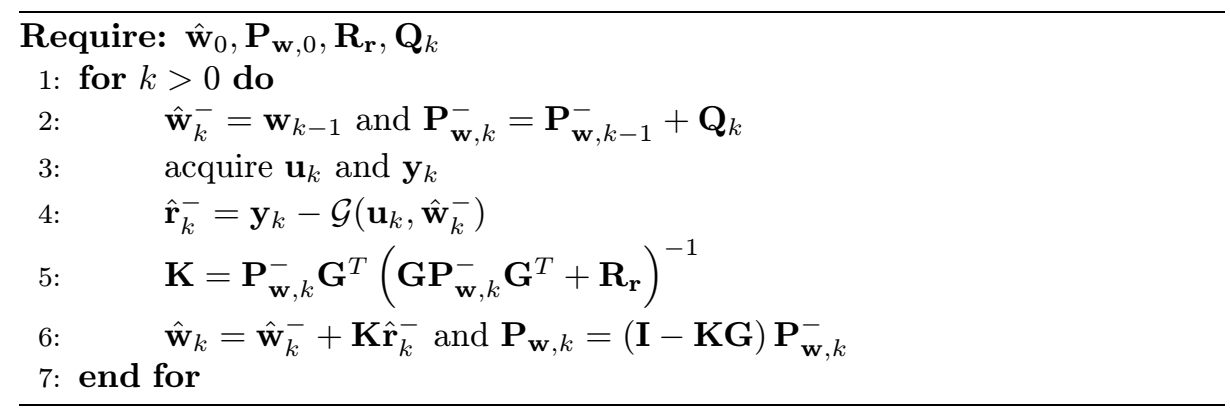

Figure 5 Algorithm 1: semilinear Kalman filter

the gain matrix can be rewritten in a more efficient way:

$$
\begin{aligned}
\mathbf{K}=\left[\left(\mathbf{P}_{\mathbf{w}, k}^{-}\right)^{-1}+\mathbf{G}^{T} \mathbf{R}_{\mathbf{r}}^{-1} \mathbf{G}\right]^{-1} \mathbf{G}^{T} \mathbf{R}_{\mathbf{r}}^{-1} & \\
& =\mathbf{P}_{\mathbf{w}, k}^{-} \mathbf{G}^{T}\left[\mathbf{G} \mathbf{P}_{\mathbf{w}, k}^{-} \mathbf{G}^{T}+\mathbf{R}_{\mathbf{r}}\right]^{-1}
\end{aligned}
$$

A Kalman filter applied to the update rule for the health parameters (6) is represented in Fig. 4. Algorithm 1 (Fig. 5) summarizes in a pseudocode style the predictor-corrector architecture of the Kalman filter.

\section{Prediction step:}

- the estimation of the health parameters at instant $k-1$ is provided by the transition model (4) which gives an a priori value $\hat{\mathbf{w}}_{k}^{-}$and an a priori covariance matrix $\mathbf{P}_{\mathbf{w}, k}^{-}$(line 2);

- the data are acquired (line 3);

- the a priori value of the health parameters is provided to the engine model which delivers an estimation of the measurements $\hat{\mathbf{y}}_{k}^{-}$; these estimated measurements are compared to the values measured on the engine and a priori residuals are formed $\hat{\mathbf{r}}_{k}^{-}=\mathbf{y}_{k}-\hat{\mathbf{y}}_{k}^{-}$(line 4).

\section{Correction step:}

- the Kalman gain $\mathbf{K}$ is computed using Eq. (8) (line 5);

- the a priori residuals are used to correct the a priori values of the health parameters using the Kalman gain matrix $\mathbf{K}$; the covariance matrix is also updated, providing the a posteriori distribution (line 6).

The Kalman gain matrix deserves a particular attention as far as the stability of the estimation procedure. The matrix $\mathbf{G P}_{\mathbf{w}, k}^{-} \mathbf{G}^{T}$ represents the projection on 
the measurements space, of the a priori covariance of the heath parameters. This allows one to weight the uncertainty on the heath parameters to the uncertainty on the measurements. The Kalman gain may be thought as a weighting factor balancing the health parameters update with the a priori knowledge:

$$
\text { Kalman gain }=\frac{a \text { priori uncertainty }}{a \text { priori uncertainty }+ \text { measurement uncertainty }} .
$$

The interested reader may consult Ref. [17] for an extensive derivation and additional details, in particular, for the update rule for the covariance matrix $\mathbf{P}_{\mathbf{w}, k}$.

\subsection{From Kalman Filtering to Robust Quadratic Programming Estimation}

One of the assumptions in the derivation of the Kalman filter is the zero-mean Gaussian nature of the measurement noise. The quadratic penalization of the residuals in the objective function (2) is derived directly from this hypothesis and makes the algorithm very sensitive to large residuals. Consequently, even a small amount of outliers can strongly deteriorate the quality of the estimation.

Robust estimation techniques aim at lowering the sensitivity with respect to large residuals by replacing the Gaussian probability density function by another noise distribution prone to outliers. Among the many candidate distributions, the so-called $\delta$-contaminated function (also known as Huber's function) has received much attention in the literature and is selected in this contribution. A detailed description of this function is beyond the scope of this paper but can be found in [18]. Basically, Huber's function consists in a Gaussian random variable contaminated by a fraction $\delta$ of outliers.

Mathematically, the linear dependancy in the residuals $\mathbf{r}_{k}$ in Eq. (5) is replaced with a function $\psi\left(\mathbf{r}_{k}\right)$ that lowers the influence of large residuals:

$$
\left(\mathbf{P}_{\mathbf{w}, k}^{-}\right)^{-1}\left(\mathbf{w}_{k}-\hat{\mathbf{w}}_{k}^{-}\right)-\mathbf{G}^{T} \mathbf{R}_{\mathbf{r}}^{-1} \psi\left(\mathbf{r}_{k}\right)=0 .
$$

Huber's weighting function is sketched in Fig. 6 for a scalar random variable $\epsilon$. Mathematically, it can be written as:

$$
\psi(\epsilon)=\max (-\Delta \sigma, \min (\epsilon, \Delta \sigma))
$$

where $\sigma$ is the standard deviation of the "clean" Gaussian variable and the scalar $\Delta$ is a threshold depending on the contamination level $\delta$ (e.g., $\Delta=1.399$ for $\delta=0.05)$.

Adopting Huber's function as the penalization function of the residuals leads to robustness against sensor faults but turns the simple linear function (5) for 


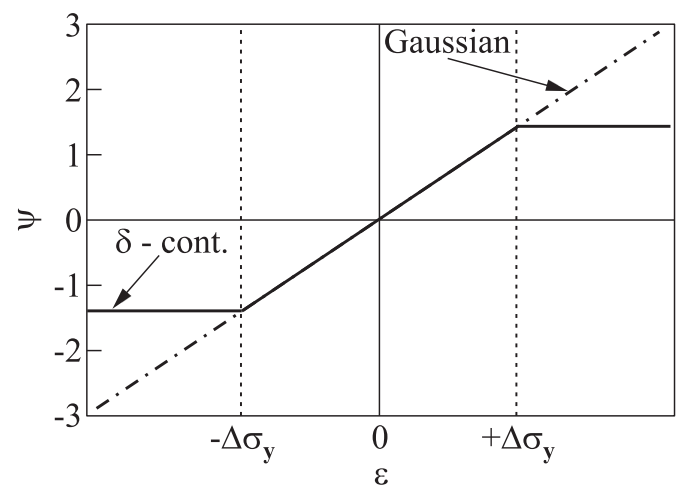

Figure 6 Huber's weighting function for a scalar

parameter update into a nonlinear one. No explicit update formula can be obtained and relation (9) must be solved numerically, with Newton's method, for instance. This might be cumbersome for on-line applications.

Nonetheless, it is shown in [12] that the nonlinear program resulting from the choice of a noise distribution following Huber's law can be transformed into a quadratic program for which efficient and fast solvers are available [19]. In [13], the authors have derived a Sensor Fault Detection and Isolation module based on this formulation which has been integrated with a tool for engine performance monitoring. As a reminder, the milestones are reported below.

As a first step, a vector $\mathbf{b}_{k}$ is introduced in the measurement Eq. (1) to model the outliers:

$$
\mathbf{y}_{k}=\mathcal{G}\left(\mathbf{u}_{k}, \mathbf{w}_{k}\right)+\mathbf{b}_{k}+\epsilon_{k}
$$

The objective function of the robust estimation problem becomes*:

$$
\mathcal{J}\left(\mathbf{w}_{k}, \mathbf{b}_{k}\right)=\frac{1}{2}\left(\mathbf{w}_{k}-\hat{\mathbf{w}}_{k}^{-}\right)^{T}\left(\mathbf{P}_{\mathbf{w}, k}^{-}\right)^{-1}\left(\mathbf{w}_{k}-\hat{\mathbf{w}}_{k}^{-}\right)+\frac{1}{2} \mathbf{r}_{k}^{T} \mathbf{R}_{\mathbf{r}}^{-1} \mathbf{r}_{k}+\Delta \sigma^{-T}\left|\mathbf{b}_{k}\right|
$$

The equivalence between solving Eq. (9) for $\mathbf{w}_{k}$ with Huber's $\psi$ function and minimizing the objective function (12) with respect to $\mathbf{w}_{k}$ and $\mathbf{b}_{k}$ is established in [12]. It should be pointed out that the criterion (12) is convex and therefore, admits a global optimum.

Finally, the quantities $\mathbf{b}_{k}$ and $\left|\mathbf{b}_{k}\right|$ are replaced with their positive and negative parts in order to transform the objective function (12) into a quadratic one:

${ }^{*}$ Note that the residuals $\mathbf{r}_{k}$ are the linear functions of $\mathbf{w}_{k}$ and $\mathbf{b}_{k}$ through Eqs. (3) and (11). 


$$
\left\{\begin{array} { c } 
{ \mathbf { b } _ { k } = \mathbf { b } _ { k } ^ { + } - \mathbf { b } _ { k } ^ { - } ; } \\
{ \mathbf { b } _ { k } | = \mathbf { b } _ { k } ^ { + } + \mathbf { b } _ { k } ^ { - } }
\end{array} \quad \text { with } \quad \left\{\begin{array}{l}
\mathbf{b}_{k}^{+}=\max \left(\mathbf{b}_{k}, 0\right) ; \\
\mathbf{b}_{k}^{-}=-\min \left(\mathbf{b}_{k}, 0\right) .
\end{array}\right.\right.
$$

With this change of variables, the objective function writes down:

$$
\begin{aligned}
\mathcal{J}\left(\mathbf{w}_{k}, \mathbf{b}_{k}^{+}, \mathbf{b}_{k}^{-}\right)=\frac{1}{2}\left(\mathbf{w}_{k}-\hat{\mathbf{w}}_{k}^{-}\right)^{T}\left(\mathbf{P}_{\mathbf{w}, k}^{-}\right)^{-1}\left(\mathbf{w}_{k}-\hat{\mathbf{w}}_{k}^{-}\right) & \\
& +\frac{1}{2} \mathbf{r}_{k}^{T} \mathbf{R}_{\mathbf{r}}^{-1} \mathbf{r}_{k}+\Delta \sigma^{-T}\left(\mathbf{b}_{k}^{+}+\mathbf{b}_{k}^{-}\right) .
\end{aligned}
$$

\subsection{Constraints Handling in the Kalman Filter and Quadratic Programming Frameworks}

In the case of condition monitoring, additional information about the health parameters is generally available. For instance, assuming that no maintenance actions are undertaken, the aerodynamic efficiencies of compressors and turbines are expected not to improve over time. These constraints actually increase in some way the a priori knowledge and should therefore be taken into account in the estimation problem.

Unfortunately, the Kalman filter algorithm does not allow such constraints to be easily integrated. Two solutions have been investigated in the literature.

On the one hand, the soft-constrained approach (see [15]) modifies the objective function (2) by adding a third term analogous to the first one which forces the a posteriori values of the health parameters to stand in some neighborhood of the constrained values. While quite easy to understand, this technique ensures the respect of the constraints only on average. Moreover, from a user's standpoint, it may turn difficult to tune the various involved parameters (such as shape and size of the neighborhood).

On the other hand, the hard-constrained approach aims at enforcing the constraints at each time step. In [14], a two-step procedure is proposed. First, a regular Kalman filter estimates the parameters, which are seen as an unconstrained solution. Then, a QP problem is solved that projects the unconstrained solution inside the constraint boundary. The constrained solution possesses a number of interesting properties that are proved in [14]. This solution looks quite appealing but makes the algorithm more complex due to the addition of the projection problem.

The QP formulation introduced in the previous section for robust estimation offers the required framework to include seamlessly constraints on the health parameters. Note that the variables $\mathbf{b}_{k}^{+}$and $\mathbf{b}_{k}^{-}$are already forced to be nonnegative through their very definition, cf. Eq. (13). Similarly, side constraints or even linear inequality constraints - can be set on the health parameters. In the present application, only side constraints are considered. Bounds can be 
specified either for the parameter itself, or for its derivative as shown for the case of upper bounds in:

$$
\begin{aligned}
& \mathbf{w}_{k}(j) \leq \mathbf{w}^{\mathrm{up}}(j) ; \\
& \mathbf{w}_{k}(j) \leq \hat{\mathbf{w}}_{k-1}^{-}(j)+\delta \mathbf{w}^{\mathrm{up}}(j)=\mathbf{w}_{k}^{\mathrm{up}}(j)
\end{aligned}
$$

where $j$ is the index of the constrained health parameter, $\mathbf{w}^{\text {up }}$ and $\delta \mathbf{w}^{\text {up }}$ are the upper bounds on the parameters and on their rates of variation, respectively.

Considering the performance tracking of a turbofan engine, it is known that the efficiency of all components and the flow capacities of the compressors decrease with usage. Accordingly, constraints of type (15) are set on the corresponding health parameters. Ideally, the bounds $\delta \mathbf{w}^{\mathrm{up}}$ on the rate of variation should be set to zero to reflect the deterioration. Nonetheless, they are set in practice to a small positive value because the estimates may oscillate around the true value.

\subsection{Practical Implementation of the Algorithm}

As a result of the inclusion of robustness and constraints in the estimation problem, the updated parameters are obtained by solving the following quasiunconstrained Quadratic Problem:

$$
\min _{\mathbf{w}_{k}, \mathbf{b}_{k}^{+}, \mathbf{b}_{k}^{-}} \mathcal{J}\left(\mathbf{w}_{k}, \mathbf{b}_{k}^{+}, \mathbf{b}_{k}^{-}\right) \quad \text { subject to }\left\{\begin{aligned}
\mathbf{b}_{k}^{+} & \geq 0 \\
\mathbf{b}_{k}^{-} & \geq 0 \\
\mathbf{w}_{k}(j) & \leq \mathbf{w}_{k}^{\text {up }}(j)
\end{aligned}\right.
$$

where $j \in$ the set of constrained parameters.

The Kalman filter structure serves as a basis in order to derive a recursive algorithm based on the QP formulation. It can be seen in algorithm 1 that the

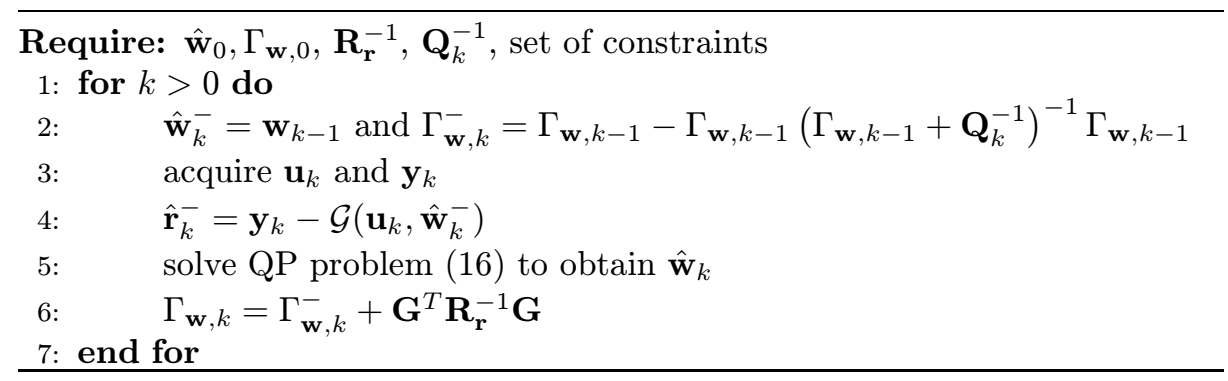

Figure 7 Algorithm 2: recursive QP estimation procedure 
Kalman filter propagates the mean and the covariance matrix of the health parameter distribution. Yet, it can also be noted that in the objective function (14), the inverse of the covariance matrix appears. Therefore, the developed recursive QP algorithm rather updates this inverted covariance matrix, also known as the information matrix, $\Gamma_{\mathbf{w}, k}=\mathbf{P}_{\mathbf{w}, k}^{-1}$ (see [20] for further details).

The pseudocode of the resulting algorithm is proposed in algorithm 2 (Fig. 7). It can be noticed that the computation of the a priori information matrix requires a matrix inversion (line 2) while the update of the information matrix is a simple matrix addition (line 6). As previously mentioned, the explicit update rule for the health parameters is replaced with the solution of Eq. (16).

\section{APPLICATION EXAMPLE}

\subsection{Engine Layout}

The application considered as a test-case is a large bypass ratio, mixed-flow turbofan. The engine performance model has been developed in the frame of the OBIDICOTE* project and is detailed in [21]. A schematic of the engine is sketched in Fig. 8 where the location of the eleven health parameters and the station numbering are also indicated. One command variable, namely, the fuel flow rate fed in the combustor, is considered in the following.

The sensor suite selected for tracking the performance degradation is representative of the instrumentation available on-board contemporary turbofan engines and is detailed in Table 1 where the accuracy of each sensor is also reported. The upper four sensors define the operating conditions (fuel flow, inlet total pressure and temperature, ambient pressure) and the remaining eight are the gas path sensors. In this configuration, the number of health parameters to estimate exceeds the number of gas path sensors. Therefore, if a sensor fault occurs, it is likely that several combinations of the health parameter deviations exactly reproduce the sensor fault, thus leading to a wrong diagnosis of the engine condition. To allow the simultaneous detection of sensor and component faults, the redundancy must consequently be increased.

In the present application, the redundancy is extended by taking into account the fact that, on modern engines, the instrumentation is generally "dualchanneled:" each of the eight probes is connected to two independent lanes (sensing element and signal processing hardware). As a result, 16 measurements are available which provides the necessary redundancy to perform the robust estimation problem. It must be pointed out that this trick does not modify the

*A Brite-Euram project for On-Board Identification, DIagnosis and COntrol of Turbofan Engine. 


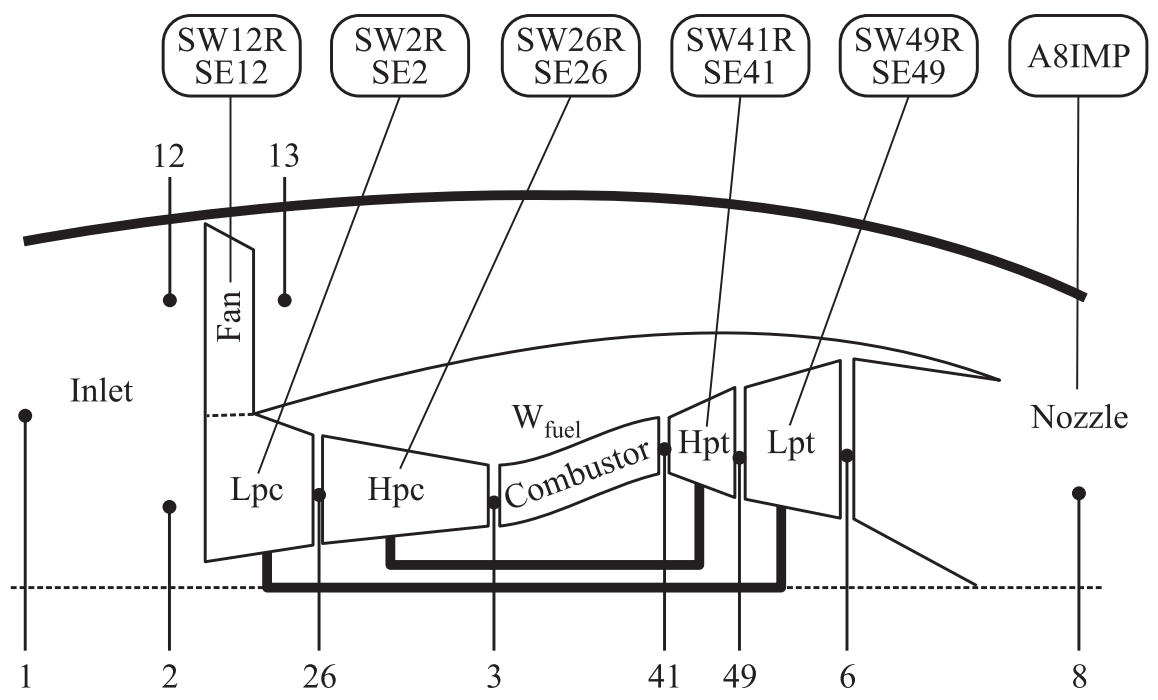

Figure 8 Turbofan layout with health parameters location

Table 1 Sensor configuration (uncertainty is three times the standard deviation)

\begin{tabular}{cc||cc}
\hline Label & Uncertainty & Label & Uncertainty \\
\hline$W_{\text {fuel }}$ & $\pm 2 \mathrm{~g} / \mathrm{s}$ & $T_{1}^{0}$ & $\pm 2 \mathrm{~K}$ \\
$p_{1}^{0}$ & $\pm 100 \mathrm{~Pa}$ & $p_{\text {amb }}$ & $\pm 100 \mathrm{~Pa}$ \\
\hline$p_{13}^{0}$ & $\pm 100 \mathrm{~Pa}$ & $T_{26}^{0}$ & $\pm 2 \mathrm{~K}$ \\
$p_{26}^{0}$ & $\pm 500 \mathrm{~Pa}$ & $T_{3}^{0}$ & $\pm 2 \mathrm{~K}$ \\
$p_{3}^{0}$ & $\pm 5000 \mathrm{~Pa}$ & $T_{6}^{0}$ & $\pm 2 \mathrm{~K}$ \\
$N_{l p}$ & $\pm 6 \mathrm{RPM}$ & $N_{h p}$ & $\pm 12 \mathrm{RPM}$ \\
\hline
\end{tabular}

observability of the health parameters, which is linked to the variety in the sensor suite.

\subsection{Definition of the Test-Cases}

A series of test-cases has been designed to assess the efficiency of the new robust and constrained estimation technique. Due to nonavailability of real data, simulated ones are used instead. Cruise conditions (Alt $=10800 \mathrm{~m}$, Mach number $\left.=0.82, \Delta T_{\mathrm{ISA}}=0 \mathrm{~K}\right)$ are assumed. The flight sequence is $5000 \mathrm{~s}$ long and 
Table 2 Description of sensor faults

\begin{tabular}{clc}
\hline Case & Description & Magnitude \\
\hline a & $5 \%$ impulsive noise & $+10 \sigma$ \\
b & $5 \%$ impulsive noise & $-10 \sigma$ \\
c & sensor bias & $+5 \sigma$ \\
d & sensor bias & $-5 \sigma$ \\
e & sensor drift & $+10 \sigma$ \\
f & sensor drift & $-10 \sigma$ \\
\hline
\end{tabular}

the sampling rate is set to $2 \mathrm{~Hz}$. Gaussian noise, whose magnitude is specified in Table 1 is added to the generated measurements to make them closer to real ones.

The engine wear is simulated from the component fault case proposed in [22]. It consists in a drift of nearly all health parameters, starting from a healthy engine (all parameters at their nominal values) at $t=0 \mathrm{~s}$ and with the following degradation at the end of the sequence $(t=5000 \mathrm{~s}):-1.5 \%$ on SW12R, $-1.2 \%$ on SE12, $-1.0 \%$ on SW2R, $-1.0 \%$ on SE2, $-2.3 \%$ on SW26R, $-1.4 \%$ on SE26, $+0.88 \%$ on SW41R, $-1.6 \%$ on SE41, and $-1.3 \%$ on SE49.

Three types of sensor faults are investigated in the test-cases: impulsive noise (spikes), sensor bias, and sensor drift. The sign of the sensor fault must also be accounted for, this results in six types of sensor faults which are summarized in Table 2 .

The level of impulsive noise per sensor is set to $5 \%$, which means that on average, 5 out of 100 samples are aberrant. The magnitude is set to \pm 10 times the standard deviation. For the biases, only one sensor is simulated faulty at a time. The bias starts at $t=1000 \mathrm{~s}$ and does not evolve till the end of the sequence. Considering the drifts, only one sensor is simulated faulty at a time as well. The drift starts at time $t=1000 \mathrm{~s}$ with a magnitude of zero and reaches a magnitude of \pm 10 standard deviation at $t=5000 \mathrm{~s}$. The magnitudes of the sensor faults have been selected according to contributions from members of the OBIDICOTE project.

\subsection{Figure of Merit}

It is proposed to compare the efficiency of three estimation tools which are the standard Kalman filter (KF), the robust Quadratic Programming (QPR) and the robust and constrained Quadratic Programming (QPRC). To this end, a figure of merit consisting in the maximum Root Mean Squared Error (RMSE) over the whole sequence is defined as: 


$$
e_{\mathrm{RMS}}=\max \left(\sqrt{\frac{1}{n} \sum_{k=1}^{n}\left(\frac{\mathbf{w}_{k}-\hat{\mathbf{w}}_{k}}{\mathbf{w}^{h l}}\right)^{2}}\right)
$$

where $\mathbf{w}^{h l}$ are the nominal values of the health parameters.

Given the stochastic nature of the measurement noise, each test-case has been run twenty times and the RMSEs reported in Tables 3-5 are the averages over those twenty runs. A test-case characterized by an averaged maximum RMSE below $0.25 \%$ is declared as successful which is indicated by a checkmark. This threshold corresponds to three times the standard deviation of the identified health parameters (i.e., the square root of the diagonal terms of the covariance matrix $\left.\mathbf{P}_{\mathbf{w}, k}\right)$.

\subsection{Results - Impulsive Noise}

In Table 3, the figures of merit are reported for the test-cases involving no sensor fault and impulsive noise (fault types a and b). The three algorithms achieve roughly the same accuracy when none of the sensors is faulty. It can be seen that the standard Kalman filter is unable to correctly track the engine condition when spikes pollute the data. This underlines the high sensitivity of least-square-based methods with respect to outliers. On the contrary, the robust QP algorithm, constrained or not, is not perturbed by the impulsive noise. For the present case, the inclusion of constraints on the health parameters does not improve the estimation.

\subsection{Results - Sensor Biases}

The figures of merit of the three algorithms in the case of a biased sensor are given in Table 4. It can be noticed that the Kalman filter is unable to perform an accurate diagnosis of the engine condition when only one of the sixteen sensors is biased. This is a further illustration of the sensitivity of the Kalman filter to aberrant data. Considering both robust tools, they seem unaffected by the biased sensor and have nearly the same efficiency as for the no-fault case. The proposed robust estimation approach allows indeed the detection and isolation of the faulty sensor which results in an accurate assessment of the engine health.

Here again, the QPRC algorithm does not perform better than the unconstrained one (QPR). This can be explained by the fact that the robust criterion has a classification nature (see Huber's function (10) and Fig. 6) and therefore, it isolates sensor biases quite naturally. The sensor fault being correctly identified, its contribution is removed from the estimation process. As a result, adding constraints on the health parameters does not provide any supplemental advantage. 
Table 3 Comparison of the figure of merit $e_{\mathrm{RMS}}$ in the case of no sensor fault (nosf) and impulsive noise

\begin{tabular}{c||cc|cc|cc}
\hline Fault case & \multicolumn{2}{||}{ KF, \% } & \multicolumn{2}{c|}{ QPR, \% } & \multicolumn{2}{c}{ QPRC, \% } \\
\hline nosf & 0.08 & $\checkmark$ & 0.10 & $\checkmark$ & 0.09 & $\checkmark$ \\
a & 0.78 & - & 0.10 & $\checkmark$ & 0.10 & $\checkmark$ \\
b & 0.59 & - & 0.09 & $\checkmark$ & 0.10 & $\checkmark$ \\
\hline
\end{tabular}

Table 4 Comparison of the figure of merit $e_{\mathrm{RMS}}$ in the case of sensor biases

\begin{tabular}{c||cc|cc|cc||cc|cc|cc}
\hline \multirow{2}{*}{$\begin{array}{c}\text { Faulty } \\
\text { sensor }\end{array}$} & \multicolumn{5}{c||}{ case c: $+5 \sigma$ bias } & \multicolumn{5}{c}{ case d: $-5 \sigma$ bias } \\
\cline { 2 - 11 } & KF, \% & QPR, \% & QPRC, $\%$ & KF, \% & QPR, \% & QPRC, \% \\
\hline$p_{13}^{0}$ & 0.71 & - & 0.10 & $\checkmark$ & 0.08 & $\checkmark$ & 0.68 & - & 0.10 & $\checkmark$ & 0.11 & $\checkmark$ \\
$p_{26}^{0}$ & 0.82 & - & 0.09 & $\checkmark$ & 0.08 & $\checkmark$ & 0.62 & - & 0.11 & $\checkmark$ & 0.11 & $\checkmark$ \\
$T_{26}^{0}$ & 1.91 & - & 0.14 & $\checkmark$ & 0.12 & $\checkmark$ & 1.84 & - & 0.10 & $\checkmark$ & 0.09 & $\checkmark$ \\
$p_{3}^{0}$ & 0.76 & - & 0.10 & $\checkmark$ & 0.08 & $\checkmark$ & 0.63 & - & 0.10 & $\checkmark$ & 0.07 & $\checkmark$ \\
$T_{3}^{0}$ & 0.69 & - & 0.10 & $\checkmark$ & 0.08 & $\checkmark$ & 0.69 & - & 0.10 & $\checkmark$ & 0.08 & $\checkmark$ \\
$N_{l p}$ & 0.76 & - & 0.09 & $\checkmark$ & 0.09 & $\checkmark$ & 0.62 & - & 0.10 & $\checkmark$ & 0.08 & $\checkmark$ \\
$N_{h p}$ & 0.76 & - & 0.10 & $\checkmark$ & 0.08 & $\checkmark$ & 0.61 & - & 0.12 & $\checkmark$ & 0.08 & $\checkmark$ \\
$T_{6}^{0}$ & 0.86 & - & 0.10 & $\checkmark$ & 0.08 & $\checkmark$ & 0.55 & - & 0.12 & $\checkmark$ & 0.08 & $\checkmark$ \\
\hline
\end{tabular}

Table 5 Comparison of the figure of merit $e_{\mathrm{RMS}}$ in the case of sensor drifts

\begin{tabular}{c||cc|cc|cc||cc|cc|cc}
\hline \multirow{2}{*}{$\begin{array}{c}\text { Faulty } \\
\text { sensor }\end{array}$} & \multicolumn{4}{c||}{ case e: $+10 \sigma$ drift } & \multicolumn{5}{c}{ case f: $-10 \sigma$ drift } \\
\cline { 2 - 10 } & KF, \% & QPR, \% & QPRC, \% & KF, \% & QPR, \% & QPRC, \% \\
\hline$p_{13}^{0}$ & 1.27 & - & $\mathbf{1 . 1 8}$ & - & 0.09 & $\checkmark$ & 0.70 & - & 0.10 & $\checkmark$ & 0.10 & $\checkmark$ \\
$p_{26}^{0}$ & 0.98 & - & 0.10 & $\checkmark$ & 0.08 & $\checkmark$ & 1.09 & - & 0.20 & $\checkmark$ & 0.14 & $\checkmark$ \\
$T_{26}^{0}$ & 3.59 & - & 0.21 & $\checkmark$ & 0.14 & $\checkmark$ & 3.47 & - & 0.14 & $\checkmark$ & 0.10 & $\checkmark$ \\
$p_{3}^{0}$ & 0.84 & - & 0.10 & $\checkmark$ & 0.08 & $\checkmark$ & 1.00 & - & 0.09 & $\checkmark$ & 0.07 & $\checkmark$ \\
$T_{3}^{0}$ & 0.80 & - & 0.10 & $\checkmark$ & 0.08 & $\checkmark$ & 0.92 & - & 0.09 & $\checkmark$ & 0.08 & $\checkmark$ \\
$N_{l p}$ & 0.82 & - & 0.10 & $\checkmark$ & 0.08 & $\checkmark$ & 0.94 & - & 0.10 & $\checkmark$ & 0.10 & $\checkmark$ \\
$N_{h p}$ & 0.80 & - & 0.10 & $\checkmark$ & 0.07 & $\checkmark$ & 1.56 & - & $\mathbf{0 . 9 4}$ & - & 0.14 & $\checkmark$ \\
$T_{6}^{0}$ & 1.14 & - & 0.10 & $\checkmark$ & 0.07 & $\checkmark$ & 1.16 & - & $\mathbf{0 . 6 8}$ & - & 0.10 & $\checkmark$ \\
\hline
\end{tabular}




\subsection{Results - Sensor Drifts}

The performance of the diagnosis tools when the data is contaminated by a drifting reading on one sensor is presented in Table 5. As for the sensor bias cases, the regular Kalman filter is totally fooled by the drifting sensor and the resulting health assessment is spoiled. It can also be seen that the QPR algorithm provides an accurate estimation in all but three cases which are a positive drift on fan bypass outlet pressure $p_{13}^{0}$, and a negative drift on high pressure spool speed $N_{l p}$ and on exhaust gas temperature $T_{6}^{0}$. For those three cases, the QPR algorithm erroneously assigns the sensor fault to the healthy lane. As a result, the health parameters are adapted so as to cope with the real engine condition and with the faulty sensor channel. This misclassification of the sensor fault is due to its nature. Indeed, the drift starts with a null magnitude and increases linearly with a soft slope. Hence, in the early moments after it appears, the drift
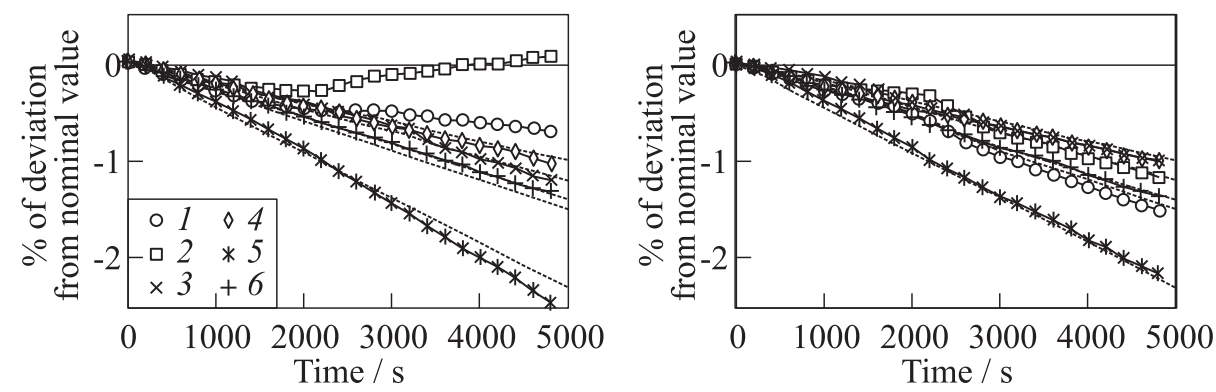

(a)
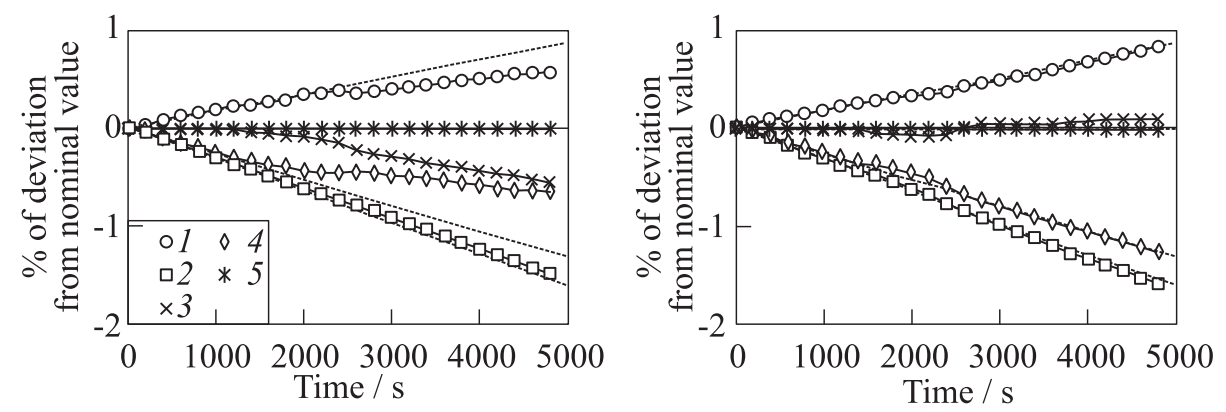

(b)

Figure 9 Identification of the health parameters by the QPR (left) and QPRC (right) algorithms with a positive drift on $p_{13}^{0}-$ dotted lines show actual parameter values: (a) 1 - SW12R; 2 - SE12; 3 - SW2R; 4-SE2; 5 - SW26R; and 6-SE26; and (b) 1 - SW41R; 2 - SE41; 3 - SW49R; $4-$ SE49; and 5 - ABIMP 
has a magnitude lower than the threshold of the robust criterion. Moreover, classical Gaussian noise is superimposed to this drift which makes the detection task even more difficult.

Correct isolation of every sensor drift might be achieved by enhancing the variety in the sensor suite. Another solution is to increase the available information by specifying constraints on the health parameters as has been discussed in a previous section. The figures of merit related to the QPRC algorithm in effect show that it is a wise solution which allows all test-cases to be accurately solved.

To conclude this short analysis of the results, Fig. 9 sketches the evolution of the identified health parameters in the case of a drift on $p_{13}^{0}$. The left column is related to the QPR algorithm while the right column is related to the QPRC algorithm. Figure $9 a$ shows the parameters of the compressors and Fig. $9 b$, the parameters of the turbines and nozzle.
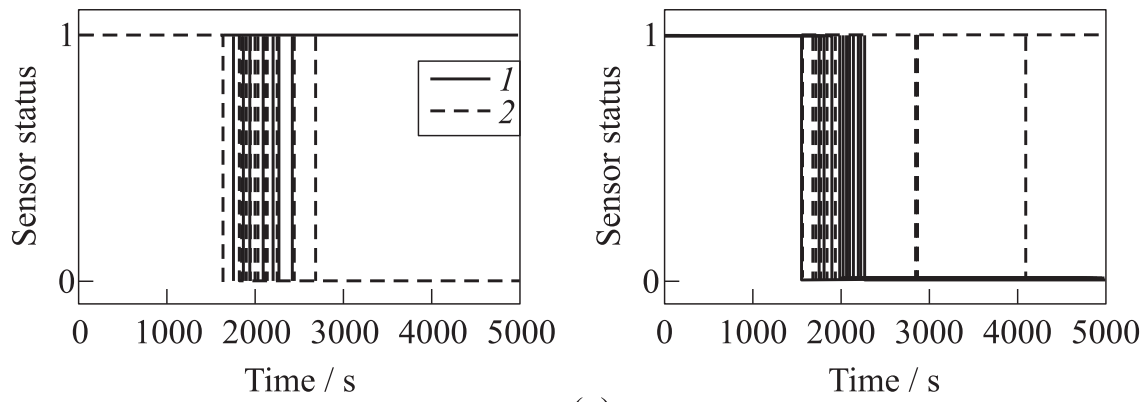

(a)
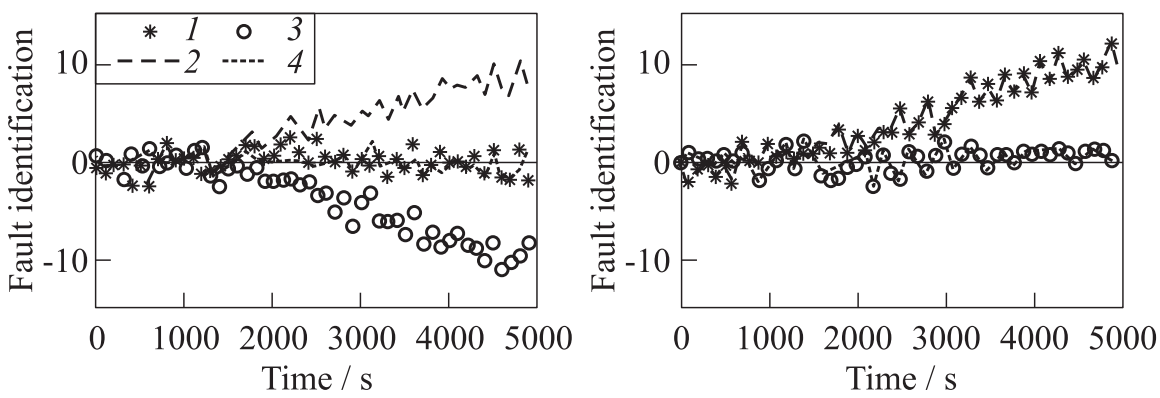

(b)

Figure 10 Identification of the drifting sensor by the QPR (left) and QPRC (right) algorithms with a positive drift on $p_{13}^{0}$ : (a) 1 - channel 1; and 2 - channel 2; and (b) 1 - channel 1 , estimations; 2 - channel 1, true; 3 - channel 2, estimations; and 4 - channel 2, true 
Figure 10 sketches the evolution of the identified drifting sensor for the same case of a drift on $p_{13}^{0}$. Figure $10 a$ shows the sensor status (good $=1$, faulty $=0$ ) and Fig. 10b, the value of the sensor fault.

Looking at the QPR results, it can be noticed that the misclassification of the drifting sensor (channel 2 is declared faulty by the algorithm whereas it is actually channel 1) leads to an error in the estimation of all parameters and particularly SE12, SW41R, SW49R, and SE49. It is also interesting to point out that the fan efficiency factor SE12 improves from about $2000 \mathrm{~s}$ which is not a physical solution.

The addition of constraints on the rate of variation on all efficiency factors and on the compressor flow factors solves this issue as can be seen from the QPRC results. Around $2000 \mathrm{~s}$, the slope of SE12 and SE49 decreases as in the unconstrained case. However, the constraints limit this effect which eases the identification of the true faulty sensor (channel 1). Then, the estimated health parameters track their actual values. A similar analysis can be conducted for the case of a drift on $N_{l p}$ and on $T_{6}^{0}$.

\section{CONCLUDING REMARKS}

In this contribution, an original algorithm for constrained and robust estimation of engine condition has been proposed based on a QP approach. The proposed methodology can be considered as a generalization of the Kalman filter which brings robustness against sensor faults and allows constraints to be set easily on the estimated parameters. The improvement in estimation efficiency have been illustrated on a number of simulated but realistic test-cases. The robust estimation tool can cope accurately with impulsive noise and sensor biases. By adding constraints which are physically meaningful, the robust and constrained estimation tool is made insensitive to sensor drifts.

Those results are promising, yet a number of issues have to be addressed in future research. The first question of practical interest is to determine the minimum level of bias for each sensor that can be effectively isolated by the robust algorithm. The second point is to make the present algorithm, which is primarily dedicated to performance monitoring, capable to track abrupt variation of the engine condition. This is a challenging problem as an abrupt component fault could be interpreted as a combination of sensor biases.

\section{REFERENCES}

1. Volponi, A. J. 2003. Foundation of gas path analysis (Part I and II). Von Karman Institute LS03-01: Gas Turbine Condition Monitoring and Fault Diagnosis. 
2. Liu, H., S. Shah, and W. Jiang. 2004. On-line outlier detection and data cleaning. Comput. Chem. Eng. 28(9):1635-47.

3. Surrender, V., and R. Ganguli. 2004. Adaptive Myriad filter for improved gas turbine condition monitoring using transient data. ASME Paper No. GT200453080 .

4. Kobayashi, T., and D.L. Simon. 2001. A hybrid neural network-genetic algorithm technique for aircraft engine performance diagnostics. AIAA Paper No. 20013763.

5. Volponi, A. J., H. DePold, R. Ganguli, and C. Daguang. 2003. The use of Kalman filter and neural network methodologies in gas turbine performance diagnostics: A comparative study. ASME J. Eng. Gas Turbines Power 125:917-24.

6. Kamboukos, Ph., and K. Mathioudakis. 2006. Multipoint non-linear method for enhanced component and sensor malfunction diagnosis. ASME Paper No. GT200690451.

7. Kobayashi, T., and D. L. Simon. 2003. Application of a bank of Kalman filters for aircraft engine fault diagnostics. ASME Paper No. GT2003-38550.

8. Kobayashi, T., and D.L. Simon. 2004. Evaluation of an enhanced bank of Kalman filters for in-flight aircraft engine sensor fault diagnostics. ASME Paper No. GT2004-53640.

9. Dewallef, P., and O. Léonard. 2001. Robust measurement validation on jet engines. 4th European Conference on Turbomachinery Proceedings.

10. Grodent, M., and A. Navez. 2001. Engine physical diagnosis using a robust parameter estimation method. AIAA Paper No. 2001-3768.

11. Dewallef, P., and O. Léonard. 2003. On-line performance monitoring and engine diagnostic using robust Kalman filtering techniques. ASME Paper No. GT200338379 .

12. Fuchs, J. J. 1999. An inverse problem approach to robust regression. 14th IFAC World Congress.

13. Borguet, J.J., and O. Léonard. 2007. A sensor-fault-tolerant diagnosis tool based on a quadratic programming approach. ASME Paper No. GT2007-27324.

14. Simon, D., and D. L. Simon. 2003. Aircraft turbofan engine health estimation using constrained Kalman filtering. ASME Paper No. GT2003-38584.

15. Dewallef, P., K. Mathioudakis, and O. Léonard. 2004. On-line aircraft engine diagnostics using a soft-constrained Kalman filter. ASME Paper No. GT200453539.

16. Kalman, R.E., and R.S. Bucy. 1961. New results in linear filtering and prediction theory. Trans. of ASME, Series D. J. Basic Engineering 83:95-107.

17. Dewallef, P. 2005. Application of the Kalman filter to health monitoring of gas turbine engines: A sequential approach to robust diagnosis. Ph.D. Thesis. University of Liège.

18. Huber, P. J. 1996. Robust statistical procedures. Society for Industrial and Applied Mathematics.

19. Fletcher, R. 2000. Practical methods of optimization. Wiley. 
20. Haykin, S. 2001. Kalman filtering and neural networks. Wiley series on adaptive and learning systems for signal processing, communication and control.

21. Stamatis, A., K. Mathioudakis, J. Ruiz, and B. Curnock. 2001. Real-time engine model implementation for adaptive control and performance monitoring of large civil turbofans. ASME Paper No. 2001-GT-0362.

22. Curnock, B. 2000. Obidicote project - Work Package 4: Steady-state test cases. Technical Report DNS62433. Rolls-Royce Plc. 